This item was submitted to Loughborough's Research Repository by the author.

Items in Figshare are protected by copyright, with all rights reserved, unless otherwise indicated.

\title{
Vibrational impact of high-speed trains. I. Effect of track dynamics
}

PLEASE CITE THE PUBLISHED VERSION

http://asadl.org/jasa/resource/1/jasman/v100/i5/p3121_s1

\section{PUBLISHER}

() Acoustical Society of America

\section{VERSION}

VoR (Version of Record)

\section{LICENCE}

CC BY-NC-ND 4.0

\section{REPOSITORY RECORD}

Krylov, Victor V.. 2012. "Vibrational Impact of High-speed Trains. I. Effect of Track Dynamics". figshare. https://hdl.handle.net/2134/10044. 
This item was submitted to Loughborough's Institutional Repository (https://dspace.lboro.ac.uk/) by the author and is made available under the following Creative Commons Licence conditions.

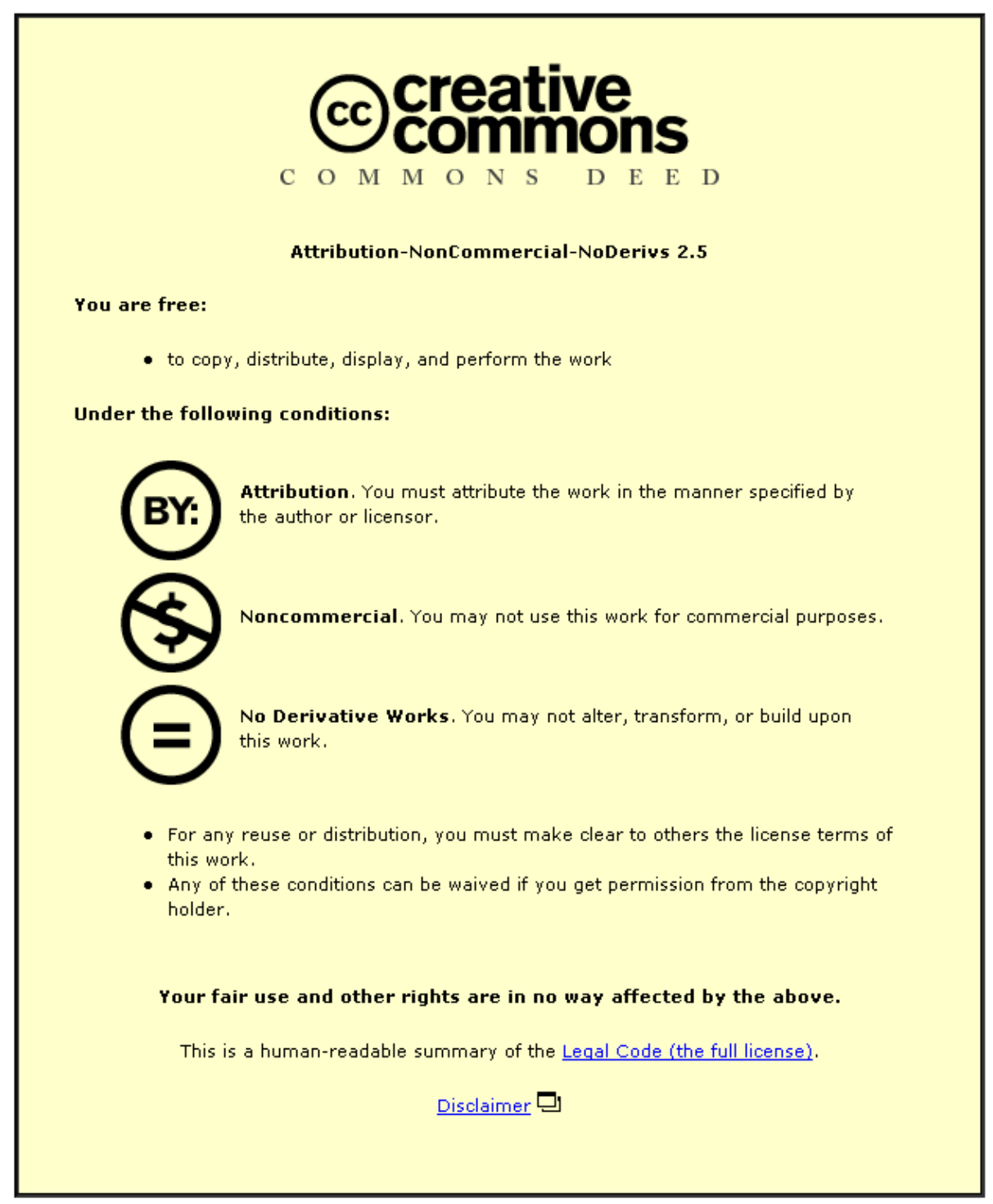

For the full text of this licence, please go to: http://creativecommons.org/licenses/by-nc-nd/2.5/ 


\title{
Vibrational impact of high-speed trains. I. Effect of track dynamics
}

\author{
Victor V. Krylov \\ Centre for Research into the Built Environment, Nottingham Trent University, Burton Street, Nottingham \\ NG1 4BU, United Kingdom
}

(Received 5 October 1995; revised 17 May 1996; accepted 24 June 1996)

\begin{abstract}
The dramatic increase in speeds of modern passenger trains makes it important to consider the vibrational impact of such trains on the built environment. In the author's earlier paper in which a quasistatic approach to the calculation of track deflection curves had been used [V. V. Krylov, Appl. Acoust. 44, 149-164 (1995)], it had been shown that a very large increase in generated ground vibration level (about $70 \mathrm{~dB}$, as compared to conventional trains) may occur if train speed exceeds the velocity of Rayleigh surface waves in the ground. Such a situation might arise, for example, with French TGV trains for which speeds over $515 \mathrm{~km} / \mathrm{h}$ have been achieved. The present paper investigates the effect of track bending waves propagating in the system track/ground on railway-generated ground vibrations. It is shown that for train speeds approaching the minimal phase velocity of bending waves the level of generated ground vibrations is reduced. Theoretical results are illustrated by numerically calculated frequency spectra of ground vibrations generated by single axle loads traveling at different speeds and by TGV or Eurostar high-speed trains. (C) 1996 Acoustical Society of America.
\end{abstract}

PACS numbers: 43.40.At, 43.40.Kd [PJR]

\section{INTRODUCTION}

In today's world, the railways have become one of the most advanced and fast developing branches of transportation. ${ }^{1,2}$ The reasons are: the relatively low air pollution per passenger, compared to motorcars, and the very high speeds achievable by the most advanced modern trains, e.g., French TGV trains for which maximum speed of more than $515 \mathrm{~km} / \mathrm{h}$ was recorded in May 1990. Prospective plans for the year 2010 assume that the New European Trunk Line will have connected Paris, London, Brussels, Amsterdam, Cologne, and Frankfurt by a high-speed railway service that will provide fast and more convenient passenger communications within Europe.

Unfortunately, the increased speeds of modern trains are likely to increase levels of associated noise and vibration that are significant even for conventional railways. ${ }^{3-10}$

Although a number of experimental and theoretical investigations of generated ground vibrations have been carried out for conventional passenger and heavy-freight trains traveling both above- and underground, ${ }^{4-10}$ very little has been done, so far, with regard to vibrations from high-speed trains. Theoretical investigations of such kind have been recently undertaken by the present author. ${ }^{10,11}$ It has been shown in Refs. 10 and 11 that high-speed trains are generally accompanied by higher levels of generated ground vibrations. An especially large increase in vibration level (more than $70 \mathrm{~dB}$, as compared to conventional trains) may occur if train speeds $\nu$ exceed the velocity of Rayleigh surface waves in the ground $c_{R}$ (such trains are called "trans-Rayleigh trains" in this paper). The condition $\nu>c_{R}$, which is similar to that of supersonic jets, can be met, e.g., by French TGV trains traveling along tracks placed on relatively soft grounds.

In the present paper, we examine the effect of one of the possible implications of the dynamic behavior of the trackthe dispersive bending waves freely propagating in the track/ ground system-on the shapes of track deflection curves and eventually on generating ground vibrations. So far, the influence of these waves has not been taken into account in the problem under consideration. ${ }^{10,11}$ This is quite true for conventional trains traveling at speeds which are much lower than critical velocities of track bending waves. However, for high-speed trains the influence of critical track-wave velocities might become essential, resulting in noticeable modification of generated ground vibration spectra.

In the following sections we describe the dynamics of track deflection as a result of impact of moving axle loads. Then we introduce the Green's function approach to the calculation of railway-generated ground vibrations and derive analytical expressions for ground vibration spectra which take into account the effect of bending waves propagating in the system track/ground. Finally, we discuss the results of the numerical calculations of ground vibration spectra generated by single-axle loads traveling at different speeds and by complete TGV or Eurostar high-speed trains.

\section{GENERATION MECHANISM}

\section{A. General remarks}

As has been demonstrated experimentally for conventional trains, ${ }^{3,4}$ there are several mechanisms of generating ground vibrations which may contribute to the total ground vibration level in different frequency bands. Among these mechanisms one can mention the wheel-axle pressure onto the track, the effects of joints in unwelded rails, the unevenness of wheels or rails (all these mechanisms cause vibrations at train-speed-dependent frequencies), and the dynamically induced forces of carriage and wheel-axle bending 
(a)

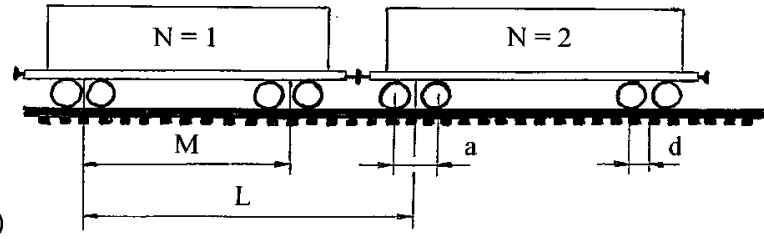

(b)

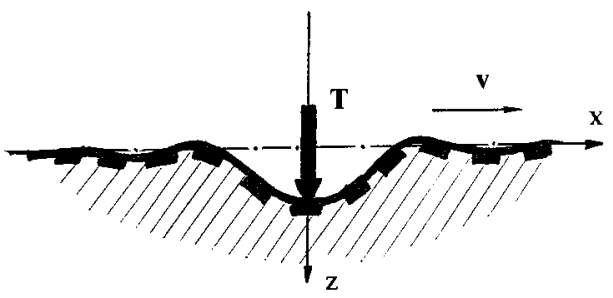

(c)

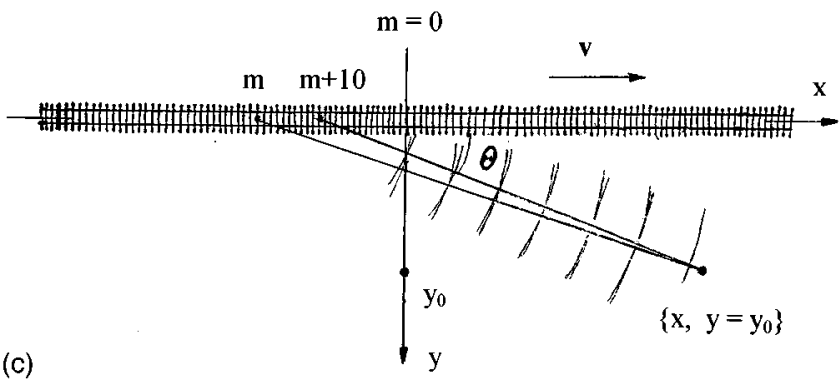

FIG. 1. (a) Geometrical parameters of track and train; (b) wheel-axle pressure mechanism of ground vibration generation; (c) superposition of ground vibrations generated by different sleepers at the point of observation $\{x, y\}$.

vibrations excited mainly by unevenness of wheels and rails (these occur at their natural frequencies). The most common generation mechanism is a pressure of wheel axles onto the track. It always persists, whereas all other mechanisms may be eliminated (at least in theory) if rails and wheels are ideally smooth and no carriage or wheel-axle bending vibrations occur. For very high-quality tracks and wheels, the wheelaxle pressure mechanism is probably a major contributor to train-speed-dependent components of the low-frequency vibration spectra (up to $50 \mathrm{~Hz}$ ), including the so called sleeper passing frequency (or simply passage frequency) $f_{p}=\nu / d$, where $\nu$ is the train speed and $d$ is the distance between sleepers. In this paper we consider contribution of the wheelaxle pressure mechanism only, assuming that rails and wheels are ideally smooth and no carriage or wheel-axle vibrations are excited.

Let us consider a train having $N$ carriages and moving at a speed $\nu$ on a perfectly welded track with a sleeper periodicity $d$ [Fig. 1(a)]. The wheel-axle pressure generation mechanism being considered results in downward deflections of the track beneath each wheel axle [Fig. 1(b)]. These deflections produce a wave-like motion along the track moving at a speed $\nu$ and result in the distribution of the axle load over the sleepers involved in the length of the track deflection bowl. ${ }^{8-11}$ Thus each sleeper acts as a vertical force applied to the ground during the time necessary for a deflection curve to pass through the sleeper. These result in the genera- tion of ground vibrations by passing trains: Since in the considered frequency band, (up to $50 \mathrm{~Hz}$ ) the characteristic wavelengths of generated elastic waves are much larger than the sleeper dimensions, each sleeper can be regarded as a point vertical force. The problem then requires superposition of the elastic fields radiated by all sleepers due to the passage of all wheel axles [Fig. 1(c)].

\section{B. Dynamic calculation of track deflection curves}

An important aspect of the above-discussed wheel-axle pressure mechanism is the calculation of the track deflection curve as a function of the elastic properties of track and soil and of the magnitude of the axle load. The form and magnitude of the deflection curve determine ground vibration frequency spectra generated by each sleeper. In turn, these spectra strongly affect the resulting ground vibration spectrum generated by a passing train.

Since the track deflection distance is usually greater than the distance between sleepers, one can ignore the influence of rail periodic support by sleepers in the problem of track deflection under the impact of a moving load. Instead we treat a track (i.e., two parallel rails with periodically fastened sleepers) as an Euler-Bernoulli elastic beam of uniform mass $m_{0}$ lying on an elastic or viscoelastic half-space $z>0$. For simplicity we assume that the uniform mass $m_{0}$ of the beam is formed entirely by the track (i.e., by rails and sleepers only), although, in practice, an adhered layer of ballast may form an additional mass which might result in the reduction of track wave velocities (see below).

For high-speed trains, which are the subject of this paper, it may happen that train speeds $\nu$ become of the same order as the minimal phase velocity $c_{\min }$ of dispersive bending waves propagating in the system track/ballast. In this case one can expect that dynamic effects associated with these waves will play a noticeable role in determining track deflection curves. To analyze such effects, one should use the dynamic equation of a beam on an elastic foundation (see, e.g., Ref. 12):

$$
E I \frac{\partial^{4} w}{\partial x^{4}}+m_{0} \frac{\partial^{2} w}{\partial t^{2}}+\alpha w=T \delta(x-\nu t) .
$$

Here $w$ is a beam deflection magnitude, $E$ and $I$ are Young's modulus and the cross-sectional momentum of the beam, $\alpha$ is the proportionality coefficient of the elastic foundation, $x$ is the distance along the beam, $T$ is a vertical point force applied to the beam at $x=\nu t$, and $\delta(x)$ is the Dirac's delta function. The coefficient $\alpha$ in (1) depends on stiffness of the ground, on stiffness of the ballast layer and ballast mats as well as of the resilient pads inserted between rails and sleepers and under sleepers. In further consideration we assume that a well-compacted ballast is always present and is largely responsible for the proportionality coefficient $\alpha$ of the equivalent Winkler foundation corresponding to the combined system ballast/ground. The results of static track deflection tests show that, e.g., for typical British rail tracks with $E I=4.85 \mathrm{MN} / \mathrm{m}^{2}$ lying on a well-compacted ballasted roadbed, the coefficient $\alpha$ is determined mainly by the ballast and is equal to $52.6 \mathrm{MN} / \mathrm{m}^{2} .^{13}$ 
It is useful first to consider free-wave propagation in the supported beam, i.e., to analyze (1) with the right-hand side equal to zero. In this case, substitution of the solution in the form of harmonic bending waves $w=A \exp (i k x-i \omega t)$ into (1) gives the following dispersion equation for track waves propagating in the system:

$$
\omega=\left(\alpha+E I k^{4}\right)^{1 / 2} / m_{0}{ }^{1 / 2} .
$$

In the quasistatic (long-wave) approximation $(k \rightarrow 0)$, the dispersion equation (2) reduces to the well-known expression for the so-called track on ballast resonance frequency: $\omega_{t b}=\alpha^{1 / 2} / m_{0}^{1 / 2}$. For the track and ballast parameters $\alpha=52.6$ $\mathrm{MN} / \mathrm{m}^{2}$ and $m_{0}=300 \mathrm{~kg} / \mathrm{m}$, this gives $F_{t b}=\omega_{t b} / 2 \pi=67 \mathrm{~Hz}$. The frequency $F_{t b}$ represents the minimal frequency of propagating track waves. It follows from (2) that the frequency-dependent velocity of track waves $c=\omega / k$ is determined by the expression

$$
c=\left(\alpha / k^{2}+E I k^{2}\right)^{1 / 2} / m_{0}{ }^{1 / 2},
$$

which shows that at $k=(\alpha / E I)^{1 / 4}$ the velocity $c$ has a minimum $c_{\min }=\left(4 \alpha E I / m_{0}^{2}\right)^{1 / 4}$. For the above-mentioned typical track and ballast parameters $c_{\min }=326 \mathrm{~m} / \mathrm{s}(1174 \mathrm{~km} / \mathrm{h})$.

The solution of (1) with the right-hand side different from zero has the form ${ }^{12}$

$$
\begin{aligned}
w= & \left(T / 8 E I \beta^{3} \delta\right) \exp (-\beta \delta|x|)[\cos (\beta \eta x) \\
& +(\delta / \eta) \sin (\beta \eta|x|)],
\end{aligned}
$$

which generalizes the corresponding static solution. ${ }^{14}$ Here $\beta=(\alpha / 4 E I)^{1 / 4}, \delta=\left(1-\nu^{2} / c_{\min }^{2}\right)^{1 / 2}$, and $\eta=\left(1+\nu^{2} / c_{\min }^{2}\right)^{1 / 2}$.

It is seen from (4) that if the train speed $\nu$ approaches the minimal phase velocity of free track waves $c_{\text {min }}$, then $\delta \rightarrow 0$ and the amplitude $w$ in (4) goes to infinity, demonstrating a resonance behavior. The infinite value of track deflection at resonance reflects limitations of the linear elastic model considered (in this paper we discuss only the case $\left.\nu<c_{\text {min }}\right)$. For typical parameters of track and ballast mentioned above, the value of $c_{\min }$ is essentially larger than even the highest train speed $(\nu=515 \mathrm{~km} / \mathrm{h})$. However, for specially designed vibro-isolated floated tracks, or for soft marshy or sandy soils in the absence of ballast, the value of $c_{\text {min }}$ may be much lower.

The forms of a deflection curve $w(x)$ calculated according to Eq. (4) for $\nu<c_{\text {min }}$ are shown in Fig. 2 for the axle load $T=100 \mathrm{kN}$ and for the values of train speed $\nu$ equal to $0,69,138,300$, and $320 \mathrm{~m} / \mathrm{s}$ (curves $\mathrm{w} 1-\mathrm{w} 5$, respectively). One can see that the curves corresponding to the first three values of train speed (i.e., up to $500 \mathrm{~km} / \mathrm{h}$ ) are almost indistinguishable. Only for train speeds approaching the minimal (critical) track wave velocity $c_{\min }=326 \mathrm{~m} / \mathrm{s}$, a significant difference occurs.

After the track deflection curve has been determined, each sleeper should be considered as a vertical concentrated force applied to the ground surface $z=0$, with time dependence determined by the passage of the deflection curve through the sleeper. For a sleeper located at $x=0$, this force may be written in the form

$$
P(t)=T\left[2 w(\nu t) / w_{\max }\right]\left(d / x_{0}\right),
$$

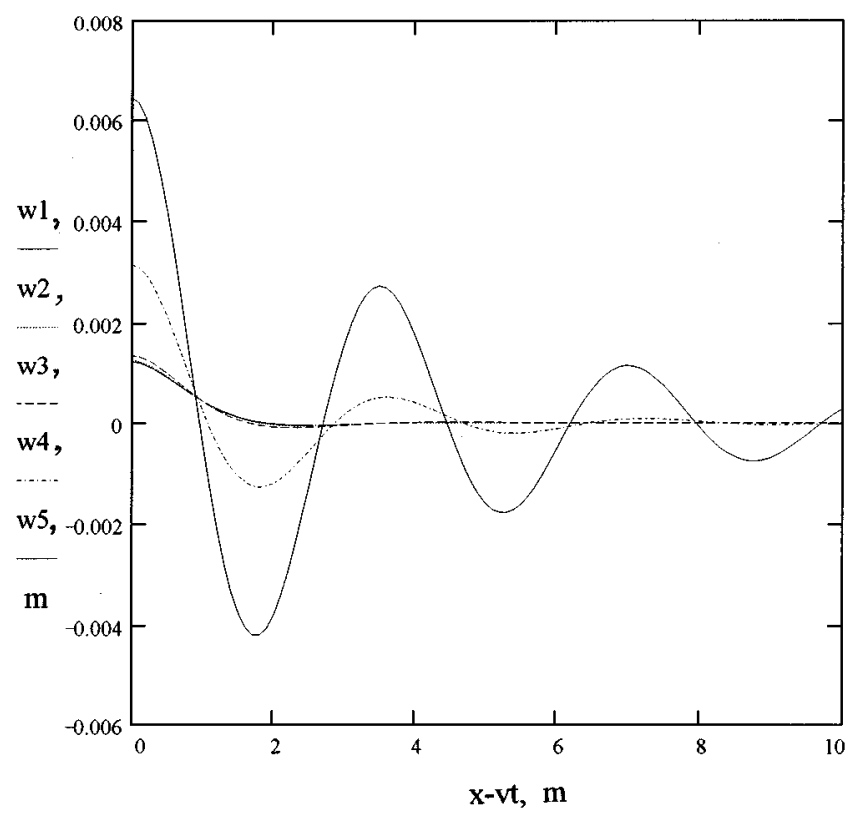

FIG. 2. Calculated track deflection curves taking into account the effect of track bending waves. Curves w1-w5 correspond, respectively, to the train speeds $0,69,138,300$, and $320 \mathrm{~m} / \mathrm{s}$; critical track wave velocity $c_{\min }$ is 326 $\mathrm{m} / \mathrm{s}$, axle load $T$ is $100 \mathrm{kN}$.

where $w_{\max }$ is the maximal value of $w(\nu t)$. Terms on the right of $T$ take into account the distribution of axle load between sleepers within the deflection curve. To derive (5), one should use the force balance equation which determines the effective number of sleepers $N_{\text {eff }}$ equalizing the applied axle load $T$ :

$$
\sum_{m=-N_{\text {eff }} / 2}^{N_{\text {eff }} / 2} \frac{T}{N_{\text {eff }}} \frac{|w(m d)|}{w_{\max }}=T,
$$

here $m$ denotes a number of a current sleeper. The numerical solution of Eq. (6) shows that for $\beta \delta$ within the range of interest (from 1 to $2 \mathrm{~m}^{-1}$ ), the value of $N_{\text {eff }}$ may be approximated by a simple analytical formula $N_{\text {eff }}=\pi / 2 \beta \delta d=x_{0} / 2 d$, which gives Eq. (5) after replacing in $w(x)$ the argument $x$ by $\nu t$.

The corresponding forces $P$ applied from each sleeper to the ground are shown in Fig. 3 as functions of $\nu t$ for the same parameters as in Fig. 2 (curves P1-P5). In all cases of essential influence of the dynamic effects, the characteristic track deflection distance, determined as $x_{0}=\pi / \beta \delta$, is increased as compared to the quasistatic case $\left(x_{0}=\pi / \beta\right){ }^{9-11}$ This results in a larger number of sleepers $N_{\text {eff }}$ involved in a deflection area $\left(N_{\text {eff }}=x_{0} / 2 d=\pi / 2 \beta \delta d\right)$ and in smaller values of the forces applied from each sleeper to the ground. In addition to this, as train speeds approach the minimal track wave velocity $c_{\min }$, these forces undergo a number of oscillations at the circular frequency $\omega=\beta \eta \nu=\beta\left(1+\nu^{2} / c_{\text {min }}^{2}\right)^{1 / 2} \nu$. This may result in distortions of generated ground vibration spectra at the upper frequency bands. 


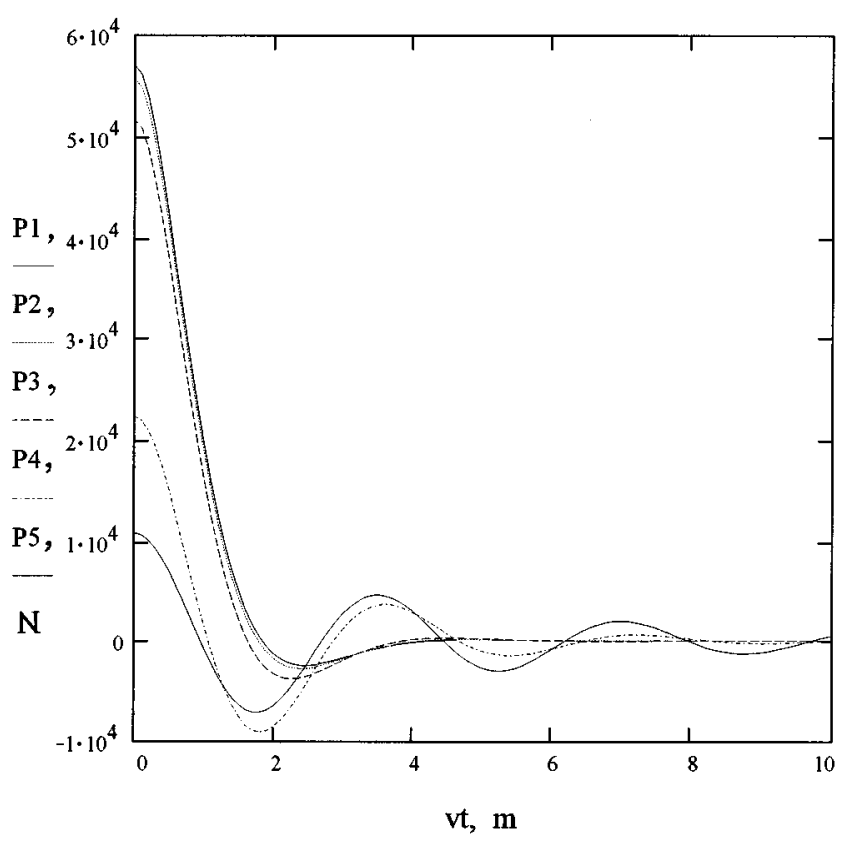

FIG. 3. Vertical forces applied from each sleeper to the ground as functions of $\nu t$ for axle loads $T=100 \mathrm{kN}$ moving along the track at speeds $0,69,138$, 300 , and $320 \mathrm{~m} / \mathrm{s}$ (curves w1-w5); distance between sleepers $d$ is $0.7 \mathrm{~m}$; all other parameters are the same as in Fig. 2.

\section{GREEN'S FUNCTION FORMALISM}

\section{A. The Green's function for an elastic half-space}

The physical meaning of the Green's function for the problem under consideration is that it describes ground vibrations generated by individual sleepers which can be regarded as point sources in the low-frequency band. To derive the corresponding Green's function, one can make use of the results from the well-known axisymmetric problem for the excitation of an elastic half-space by a vertical point force applied to the surface (at the low-frequency band considered, we neglect the influence of a thin ballast layer on elastic wave propagation). The solution of this problem gives the corresponding components of the dynamic Green's tensor (or, for simplicity, the Green's function) $G_{z i}$ for an elastic half-space. This function satisfies the dynamic equation of elasticity for a half-space and the boundary conditions on the surface. The solutions of this and related problems of an elastic half-space excitation by different load forces, whether using the terminology of Green's function or otherwise, have been considered by many authors beginning with $\mathrm{H}$. Lamb. ${ }^{15}$ Different methods of solution of the Lamb's problem, both in time and frequency domains, are reviewed in the books. ${ }^{16,17}$

In what follows, only Rayleigh surface waves (the Rayleigh part of the Green's function) are considered since Rayleigh waves transfer most of the vibration energy to remote locations. For these waves the Fourier transform of the vertical component of ground vibration velocity at $z=0$ may be written in the form (see also Ref. 9)

$$
\begin{aligned}
v_{z}(\rho, \omega) & =P(\omega) G_{z z}(\rho, \omega) \\
& =V(\omega)(1 / \sqrt{\rho}) \exp \left(i k_{R} \rho-\gamma k_{R} \rho\right),
\end{aligned}
$$

where

$$
\begin{aligned}
V(\omega)= & (\pi / 2)^{1 / 2} P(\omega)(-i \omega) q k_{R}{ }^{1 / 2} k_{t}^{2} \\
& \times \exp (-i 3 \pi / 4) / \mu F^{\prime}\left(k_{R}\right),
\end{aligned}
$$

here

$$
P(\omega)=\left(\frac{1}{2 \pi}\right) \int_{-\infty}^{\infty} P(t) \exp (i \omega t) d t
$$

is the Fourier transform of $P(t), \rho=\left[\left(x-x^{\prime}\right)+\left(y-y^{\prime}\right)^{2}\right]^{1 / 2}$ is the distance between the source (with current coordinates $x^{\prime}, y^{\prime}$ ) and the point of observation (with coordinates $x, y$ ), $\omega=2 \pi F$ is a circular frequency, $k_{R}=\omega / c_{R}$ is the wave number of a Rayleigh surface wave, $c_{R}$ is the Rayleigh wave propagation velocity, $k_{t}=\omega / c_{t}$ and $k_{l}=\omega / c_{t}$ are the wave numbers of longitudinal and shear bulk elastic waves, where $c_{l}=\left[(\lambda+2 \mu) / \rho_{0}\right]^{1 / 2}$ and $c_{t}=\left(\mu / \rho_{0}\right)^{1 / 2}$ are longitudinal and shear propagation velocities, $\lambda$ and $\mu$ are the elastic Lame' constants; $\rho_{0}$ is mass density of the ground, and $q=\left(k_{R}^{2}-k_{l}^{2}\right)^{1 / 2}$. The factor $F^{\prime}\left(k_{R}\right)$ is a derivative of the socalled Rayleigh determinant:

$$
F(k)=\left(2 k^{2}-k_{t}^{2}\right)^{2}-4 k^{2}\left(k^{2}-k_{t}^{2}\right)^{1 / 2}\left(k^{2}-k_{l}^{2}\right)^{1 / 2}
$$

taken at $k=k_{R}$, and $\gamma$ is the ground attenuation constant defined via replacing $1 / c_{R}$ in the exponential of the Green's function for ideally elastic medium by the complex value $1 / c_{R}+i \gamma / c_{R}$, where $\gamma=0.001-0.1$ describes the "strength" of dissipation of Rayleigh waves [Eq. (7) implies a linear frequency dependence of soil attenuation, in agreement with the experimental data $\left.{ }^{18-20}\right]$. The factor $1 / \sqrt{ } \rho$ in (7) describes cylindrical spreading of Rayleigh waves with propagation distance.

It is seen from (7) and (8) that the Fourier transform $P(\omega)$ plays an important role in determining spectra of radiated waves. Taking the Fourier transform of (5) and (4) and using the expression $x_{0}=\pi / \beta \delta$, one can easily obtain the corresponding formula for $P(\omega)$ which takes into account the effect of track bending waves:

$$
\begin{aligned}
P(\omega)= & \left(T \beta \delta d / \pi^{2}\right)\left\{[\beta \delta v+(\beta \eta v+\omega)] /\left[(\beta \delta v)^{2}\right.\right. \\
& \left.+(\beta \eta v+\omega)^{2}\right]+[\beta \delta v+(\beta \eta v-\omega)] /\left[(\beta \delta v)^{2}\right. \\
& \left.\left.+(\beta \eta v-\omega)^{2}\right]\right\} .
\end{aligned}
$$

In the quasistatic limit, $v / c_{\min } \rightarrow 0$ resulting in $\delta \rightarrow 0$ and $\eta \rightarrow 1$, Eq. (10) goes over to the corresponding quasistatic expression for $P(\omega){ }^{9,11}$

To describe the Fourier transform of the force applied from each sleeper to the ground for successive passage of two axle loads separated by the distance $a$ (the case of a bogie), $P_{b}(\omega)$, one should use the following obvious relationship between $P_{b}(\omega)$ and $P(\omega)^{9}$ :

$$
P_{b}(\omega)=2 P(\omega) \cos (\omega a / 2 v) .
$$

\section{B. Ground vibrations from complete trains}

To calculate the ground vibration field radiated by a complete train requires superposition of fields generated by 
each sleeper activated by all axles of all carriages, with the time and space differences between sources (sleepers) being taken into account [Fig. 1(c)].

Using the Green's function this may be written in the form $^{9-11}$

$$
v_{z}(x, y, \omega)=\int_{-\infty}^{\infty} \int_{-\infty}^{\infty} P\left(x^{\prime}, y^{\prime}, \omega\right) G_{z z}(\rho, \omega) d x^{\prime} d y^{\prime},
$$

where $P\left(x^{\prime}, y^{\prime}, \omega\right)$ describes the space distribution of all load forces acting along the track in the frequency domain. This distribution can be found by taking a Fourier transform of the time- and space-dependent load forces applied from the track to the ground.

To take account of all axles and carriages, one needs the following load forces function:

$$
\begin{aligned}
P\left(t, x^{\prime}, \quad y^{\prime}=0\right)= & \sum_{m=-\infty}^{\infty} \sum_{n=0}^{N-1} A_{n}\left[P\left(t-\left(x^{\prime}+n L\right) / v\right)\right. \\
& \left.+P\left(t-\left(x^{\prime}+M+n L\right) / v\right)\right] \\
& \times \delta\left(x^{\prime}-m d\right) \delta\left(y^{\prime}\right),
\end{aligned}
$$

here $N$ is the number of carriages, $M$ is the distance between the centers of bogies in each carriage, and $L$ is the total carriage length. Dimensionless quantity $A_{n}$ is an amplitude weight factor to account for different carriage masses. For simplicity we assume all carriage masses to be equal $\left(A_{n}=1\right)$.

Taking the Fourier transform of (13), substituting it into (12), and making simple transformations, we obtain the following expression for the frequency spectra of vertical vibrations at $z=0, x=0$, and $y=y_{0}$ generated by a moving train:

$$
\begin{aligned}
v_{z}(x= & \left.0, y=y_{0}, \omega\right) \\
= & V(\omega) \sum_{m=-\infty} \sum_{n=0}^{N-1}\left[\exp \left(-\gamma \omega \rho_{m} / c_{R}\right) / \sqrt{\rho_{m}}\right] \\
& \times[1+\exp (i M \omega / v)] \exp [i(\omega / v)(m d+n L) \\
& \left.+i\left(\omega / c_{R}\right) \rho_{m}\right] .
\end{aligned}
$$

The summation over $m$ in (13) and (14) considers an infinite number of sleepers. However, the contribution of remote sleepers is small because of soil attenuation and cylindrical spreading of Rayleigh waves, and a few hundred sleepers are adequate for practical calculations.

\section{SPECTRA OF GENERATED GROUND VIBRATIONS}

\section{A. Conventional trains}

For conventional trains $\left(v \ll c_{R}\right)$, it follows from Eq. (14) that spectra of generated ground vibrations are quasidiscrete, with the maxima at frequencies determined by the condition $(\omega / v)(m d+n L)=2 \pi l$, where $l=1,2,3, \ldots$. Obviously, $n=0$ corresponds to the passage frequencies $f_{p} s$, where $f_{p}=v / d$ and $s=1,2,3, \ldots$. Other, more frequent, maxima are determined either by the carriage length $L(m=0)$ or by a combination of both parameters (for $n \neq 0, m \neq 0$ ).
There are also many zeros present in the train vibration spectra which may be used, in practice, for suppressing vibrations from conventional trains at chosen frequencies. ${ }^{8,9}$ The most important zeros are those which do not depend on the number of sleepers or carriages and are determined only by the geometrical parameters of a carriage. For example, one of these zeros is determined by the distance $a$ between the wheel axles in a bogie [see Eq. (11) for the spectrum $P_{b}$ ]. Setting $P_{b}$ to zero, one can obtain $f_{z}=(v / a)(n+1 / 2)$ for zero frequencies. If, for instance, we want to use this condition to suppress one of the train passage frequencies $f_{p} s$, we should choose $f_{z}$ to be equal to $f_{p} s$. It follows from this that the value of $a$ should be selected as $a=(d / s)(n+1 / 2)$. Other important zero frequencies reflect the distance $M$ between bogies in a carriage. The above-written suppression condition may be applied for this case as well if to replace $a$ by $M$.

\section{B. Trans-Rayleigh trains}

The general expression (14) derived above is applicable to trains moving at arbitrary speeds. However, for the specific case of "trans-Rayleigh trains," i.e., trains traveling at speeds higher than the Rayleigh wave velocity in the ground, an additional analytical treatment is useful to elucidate the special features of the problem and to clarify the time and space distributions of radiated waves.

We first consider the vibration field generated by a single load $(N=1, M=0, L=0, a=0)$ moving at speed $v$ through a part of a track having a small number of sleepers $2 Q+1$. Let the point of observation be arbitrarily located on the ground surface, i.e., $\rho_{m}=\left[y^{2}+(x-m d)^{2}\right]^{1 / 2}$. Then, for far-field distances $\left(R \gg Q d\right.$, where $\left.R=\left[y^{2}+x^{2}\right]^{1 / 2}\right)$ the expression for $\rho_{m}$ can be simplified as follows:

$$
\rho_{m} \approx R-m d \cos \Theta,
$$

where $\cos \theta=x / R$ (here $\theta$ is the observation angle). Substitution of Eq. (15) into (14), with a limited number of sleepers being taken into account, gives the following expression for the vertical component of ground vibration velocity:

$$
\begin{aligned}
v_{z}(x, y, \omega)= & \frac{V(\omega)}{\sqrt{R}} \exp \left[(i-\gamma)\left(\omega / c_{R}\right) R\right] \\
& \times \sum_{m=-Q}^{Q} \exp [i(\omega / v) m d-(i-\gamma) \\
& \left.\times\left(\omega / c_{R}\right) m d \cos \Theta\right],
\end{aligned}
$$

where we have neglected the small term $m d \cos \theta$ in the denominator.

It is seen from (16) that maximum radiation of ground vibrations takes place if the train speed $v$ and the Rayleigh wave velocity $c_{R}$ satisfy the relation

$$
\cos \theta=c_{R} / v \text {, }
$$

which is similar to the conditions of Mach or Cherenkov radiation. This relation means that elastic fields radiated by all sleepers activated by a moving load are combined in phase at the point of observation. Since the radiation angle $\theta$ should be real $(\cos \Theta \leqslant 1)$, the train speed $\nu$ should be larger 

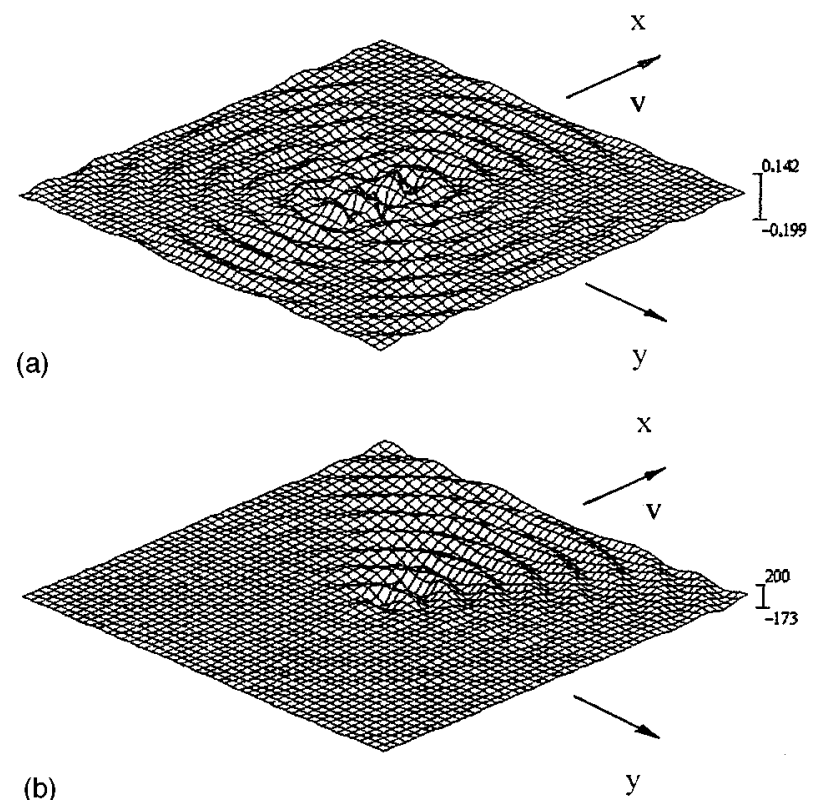

(b)

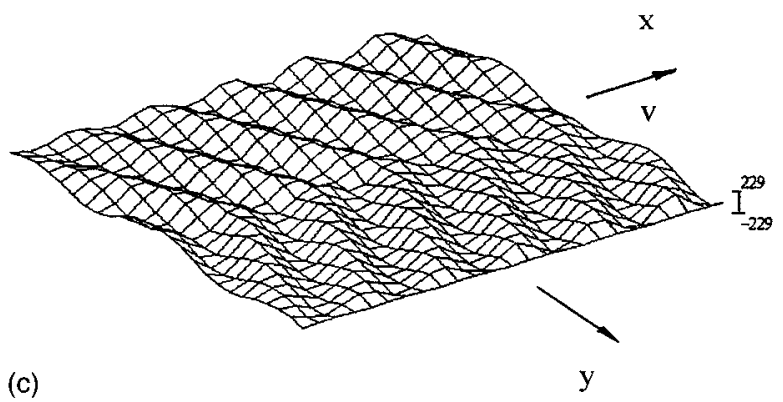

FIG. 4. Spatial distributions of ground vibration fields generated by a single-axle load moving over a small part of the track with just ten sleepers (vibration velocity or vertical surface displacement in arbitrary linear units) at the spectral component $F=31.4 \mathrm{~Hz}$ for sub-Rayleigh (a) and transRayleigh (b) speeds. (c) The corresponding spatial distribution for an axle load moving at trans-Rayleigh speed over a larger part of the track (with 100 sleepers instead of ten) is shown. The area of the ground surface considered is $48 \mathrm{~m} \times 48 \mathrm{~m}$. Mesh: $\Delta x=\Delta y=1 \mathrm{~m}$.

than Rayleigh wave velocity $c_{R}$. In this case the ground vibrations are generated as cylindrically attenuated Rayleigh surface waves [factor $(R)^{1 / 2}$ in the denominator] symmetrically propagating at angles $\Theta$ with respect to the track, and with amplitudes much larger than for "sub-Rayleigh trains."

All principal features of the above remain valid also for tracks with an infinite number of sleepers. As was shown in an earlier paper, ${ }^{9}$ dissipation of Rayleigh waves in the ground and their geometrical attenuation [factors $\rho_{m}^{1 / 2}$ in the denominator of (14)] mean that normally, only about 200 sleepers need to be considered. Since, in this case, one usually deals with the near field of radiating track, the analytical description is very bulky (like in the Fresnel zone of classical flat radiators), and it is preferable to use direct numerical calculations of formula (14) with the exact expression for the distances $\rho_{m}$.

The amplitudes of railway-generated ground vibrations for $\nu>c_{R}$ are determined by two features. The first is that under this condition the surface waves radiated by different sleepers are combined in phase. Therefore, an increase by the number of effectively radiating sleepers of the track, i.e., about 200 times, can be expected, compared to the average vibration level for conventional trains. The second feature is the dependence of the function $P(\omega)$, determined by Eq. (10), on train speed $\nu$. The analysis shows that function $P(\nu, F)$, where $F=\omega / 2 \pi$, provides an average increase of about ten times for $\nu=138.8 \mathrm{~m} / \mathrm{s}(500 \mathrm{~km} / \mathrm{h})$, compared with $\nu=13.88 \mathrm{~m} / \mathrm{s}(50 \mathrm{~km} / \mathrm{h})$. Thus a total increase of ground vibration amplitudes by $1000-2000$ times $(60-66 \mathrm{~dB})$ can be expected for the case of trans-Rayleigh trains.

It is interesting to note that, according to Eq. (16), the amplitudes of the generated vibration field radiated at angles $\Theta=\arccos \left(c_{R} / \nu\right)$ depend neither on the periodicity of sleepers $d$ nor on their number $2 Q+1$. They are determined only by the track distance considered. In fact, since the summation in (16) gives $2 Q+1$ in this case and $V(\omega)$ is proportional to $d$, the value of $\nu_{z}(x, y, \omega)$ is proportional to the distance $S=(2 Q+1) d$. Note that this dependence remains valid also for the limiting case $d \rightarrow 0$, for constant track distance $S$. This is easy to prove by replacing the sum in Eq. (16) by the integral (for simplicity we neglect the ground attenuation):

$$
\begin{aligned}
\nu_{z}(x, y, \omega)= & \lim _{d \rightarrow 0}\left\{\frac{V(\omega)}{\sqrt{R}} \frac{1}{d} \exp \left[i\left(\omega / c_{R}\right) R\right]\right\} \\
& \times \int_{-S / 2}^{S / 2} \exp [i(\omega / \nu) \xi \\
& \left.-i \cos \Theta\left(\omega / c_{R}\right) \xi\right] d \xi,
\end{aligned}
$$

where discrete distance $m d$ has been replaced by $\xi$ and sleeper spacing $d$ by differential $d \xi$. Since $V(\omega) \sim d$, we obtain that for $\cos \theta=\nu / c_{R}$, the value of $\nu_{z}(x, y, \omega)$ is proportional to $S$. This means that radiation of ground vibrations by trans-Rayleigh trains may take place also on tracks without sleepers. Note however that, for conventional low-speed trains $\left(\nu \ll c_{R}\right)$, the exponential function inside the integral in (18) oscillates quickly and for large $S$ and $\omega / \nu$ the integral value is close to zero, indicating that ground vibrations in the form of waves are almost not generated. This agrees with the well-known result of the elasticity theory ${ }^{21}$ that, for loads moving along a free surface of an elastic half-space at speed $\nu<c_{R}$, radiated wave fields do not exist (only localized quasistatic fields will accompany the moving load). Thus the presence of sleepers is essential for generating ground vibrations by conventional trains due to the mechanism of wheelaxle pressure considered here. Numerical calculations show that change of $d$ to smaller values results in noticeable reduction in high-frequency components of generated ground vibration spectra.

\section{Results of the numerical calculations}

Computation of ground vibrations generated by highspeed trains have been carried out according to Eq. (14) for different values of train speed $\nu$, track wave critical velocity $c_{\text {min }}$, number of sleepers being taken into account $2 Q+1$, and for different geometrical and physical parameters of both track and train. Summation over $m$ in Eq. (14) was carried out from $m=-Q$ to $m=Q$. In the majority of calculations the chosen value of $Q$ in Eq. (14) $(Q=150)$ was such that the 

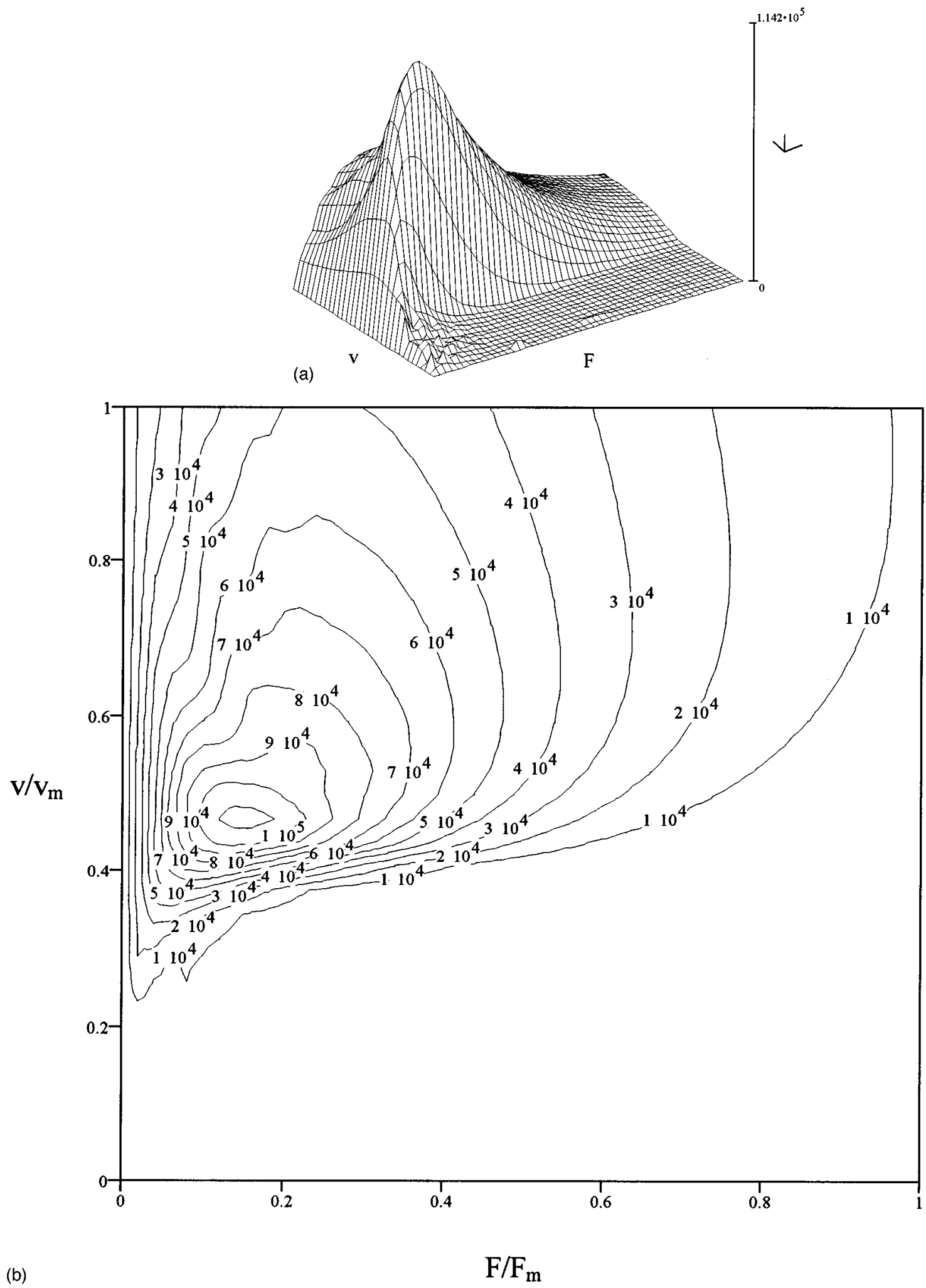

FIG. 5. Spectra of ground vibration velocity calculated using quasistatic approximation (in linear units, relative to the reference level of $10^{-9} \mathrm{~m} / \mathrm{s}$ ) for a single-axle load moving along the track at speeds from 10 to $320 \mathrm{~m} / \mathrm{s}$ (these include trans-Rayleigh speeds). The results are shown in the forms of surface (a) and contour (b) graphs for the frequency band 2-50 Hz. Mesh: $\Delta v=10 \mathrm{~m} / \mathrm{s}$ and $\Delta F=1 \mathrm{~Hz}$.

corresponding track length $(2 Q+1) d$ was greater than the total train length $N L$ and the attenuation distance of Rayleigh waves at the frequency band considered. The Poisson's ratio of soil was set at 0.25 , and the mass density of soil $\rho_{0}$ was $2000 \mathrm{~kg} / \mathrm{m}^{3}$.
In Fig. 4 the spatial distributions of ground vibration fields generated by a single moving axle load $T=100 \mathrm{kN}$ at the spectral component $F=31.4 \mathrm{~Hz}$ (vibration velocity or vertical surface displacement in arbitrary linear units) are shown for sub-Rayleigh (a) and trans-Rayleigh (b) speeds, 

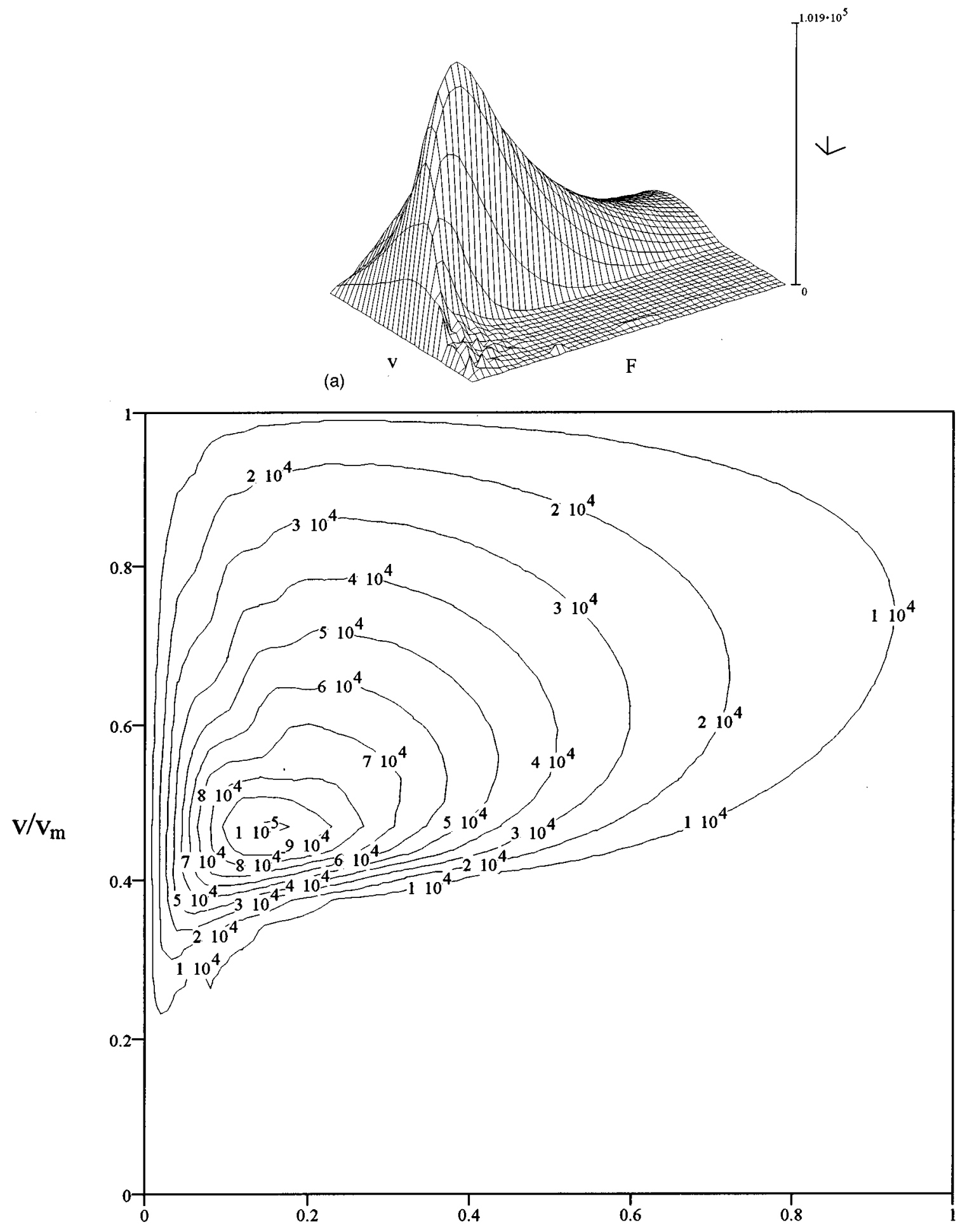

(b)

$\mathrm{F} / \mathrm{F}_{\mathrm{m}}$

FIG. 6. Spectra of ground vibration velocity calculated using the dynamic approach (in linear units, relative to the reference level of $10^{-9} \mathrm{~m} / \mathrm{s}$ ) for a single-axle load moving along the track at speeds from 10 to $320 \mathrm{~m} / \mathrm{s}$ (these include trans-Rayleigh speeds). The results are shown in the forms of surface (a) and contour (b) graphs for the frequency band 2-50 Hz. Mesh: $\Delta v=10 \mathrm{~m} / \mathrm{s}$ and $\Delta F=1 \mathrm{~Hz}$.

respectively, $\nu=50 \mathrm{~km} / \mathrm{h}$ and $\nu=500 \mathrm{~km} / \mathrm{h}$. The area of the ground surface considered is $48 \mathrm{~m} \times 48 \mathrm{~m}$. To demonstrate the formation of wave fields for both speeds, a small part of the track with just ten sleepers located in the center of the area has been considered. The Rayleigh wave velocity $c_{R}$ was set as $125 \mathrm{~m} / \mathrm{s}, \beta=1.28 \mathrm{~m}^{-1}$, and $\gamma=0$.
It is clearly seen that at low train speeds (a) the waves are radiated in almost all directions, whereas at transRayleigh speeds (b) the generated wave field is concentrated mainly in the direction of a train movement, occupying the sector roughly determined by the angles $\Theta=\arccos \left(c_{R} / \nu\right)$ with respect to the track. The amplitudes of generated waves 


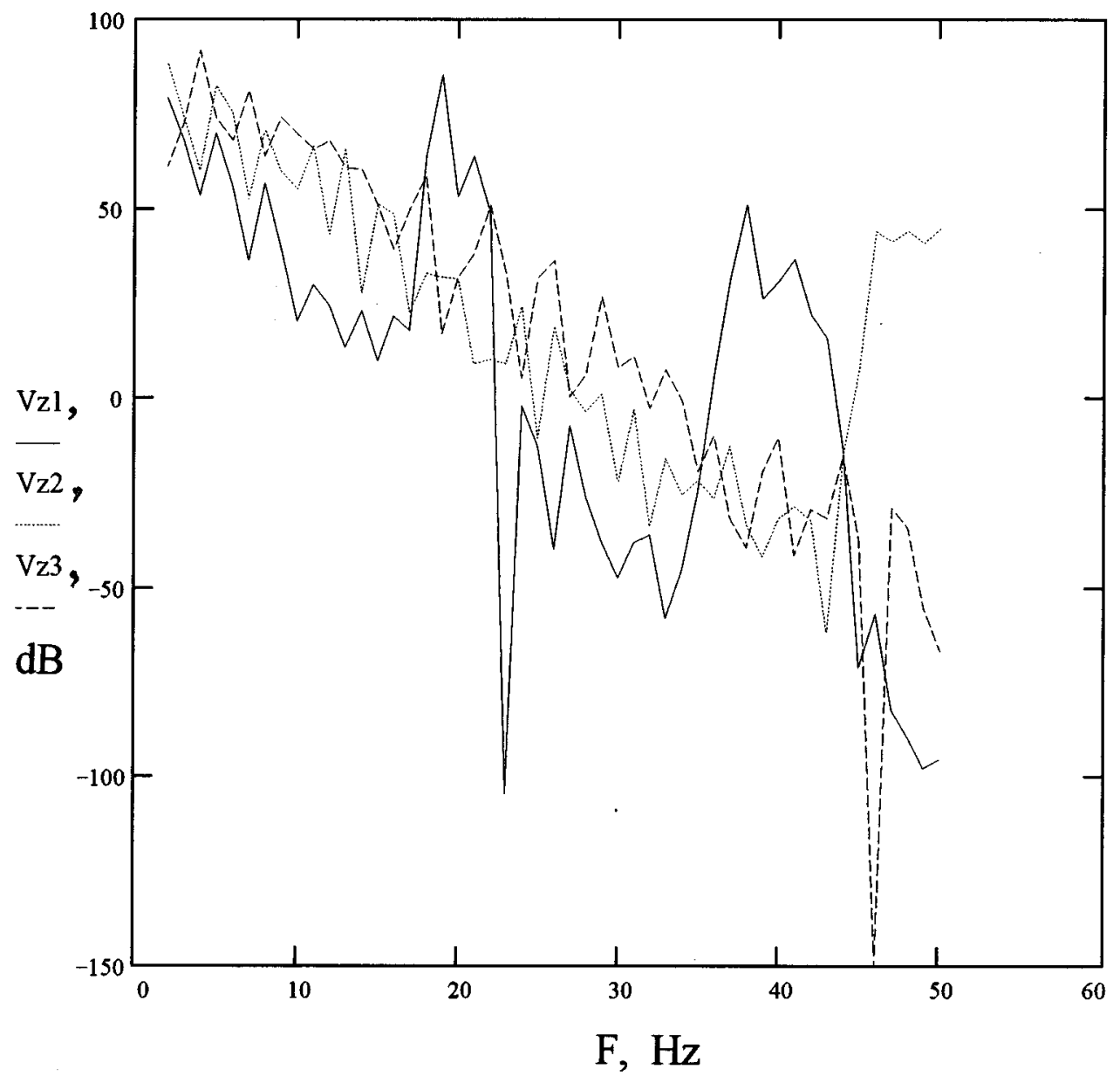

FIG. 7. Ground vibration spectra (in $\mathrm{dB}$, relative to the reference level of $10^{-9} \mathrm{~m} / \mathrm{s}$ ) generated by French TGV or Eurostar trains comprising $N=5$ equal carriages for three sub-Rayleigh values of train speed: $\nu=50 \mathrm{~km} / \mathrm{h}$ (curve Vz1), $\nu=150 \mathrm{~km} / \mathrm{h}$ (curve Vz2), and $\nu=250 \mathrm{~km} / \mathrm{h}$ (curve Vz3).

are approximately 1000 times larger in (b) than in (a) as can be seen from the vertical scales in the figures.

The spatial distribution of the ground vibration field generated by an axle load moving at trans-Rayleigh speed along a part of the track with 100 sleepers (instead of 10) is shown in Fig. 4(c). It is seen that the effect of a larger number of radiated sleepers is to produce a very high directivity of ground vibration radiation. The vibration field consists of almost perfect plain waves propagating at the angles $\Theta=\arccos \left(c_{R} / \nu\right)$ with respect to the track.

The ground vibration spectra (in linear units, relative to the reference level of $10^{-9} \mathrm{~m} / \mathrm{s}$ ) generated by a single axle load moving at speeds ranging from 10 to $320 \mathrm{~m} / \mathrm{s}$ are shown in Figs. 5 and 6, respectively, for quasistatic and dynamic calculations in the forms of surface (a) and contour (b) graphs. The value of ground attenuation was $\gamma=0.05$. The results are shown for the frequency band $2-50 \mathrm{~Hz}$; the units of calculation are $\Delta \nu=10 \mathrm{~m} / \mathrm{s}$ and $\Delta F=1 \mathrm{~Hz}$.

Comparison of Figs. 5 and 6 shows that the effects of track dynamics occur only for train speeds approaching the critical track wave velocity $\left(c_{\min }=326 \mathrm{~m} / \mathrm{s}\right)$ and reveal in reduction in spectral amplitudes of generated ground vibrations (see Fig. 6).

Ground vibration spectra generated by French TGV or Eurostar trains consisting of $N=5$ equal carriages of length
$L=18.9 \mathrm{~m}$ are shown in Fig. 7 in the frequency band $2-50$ $\mathrm{Hz}$ for three sub-Rayleigh values of a train speed: $\nu=50$ $\mathrm{km} / \mathrm{h}$ (curve Vz1), $\nu=150 \mathrm{~km} / \mathrm{h}$ (curve Vz2), and $\nu=250$ $\mathrm{km} / \mathrm{h}$ (curve Vz3). The Rayleigh wave velocity in the ground was $c_{R}=125 \mathrm{~m} / \mathrm{s}(450 \mathrm{~km} / \mathrm{h})$, and the soil attenuation coefficient has been set as $\gamma=0.05$. Since the bogies of TGV and Eurostar trains have a wheel spacing of $3 \mathrm{~m}$ and are placed between carriage ends, i.e., shared between two neighboring carriages, to use Eq. (14) one should consider each carriage as having one-axle bogies $(a=0)$ separated by the distance $M=15.9 \mathrm{~m}$. Other parameters used in the calculations were $T=100 \mathrm{kN}, \beta=1.28 \mathrm{~m}^{-1}$, and $y_{0}=30 \mathrm{~m}$.

The behavior of the curves Vz1-Vz3 shows that, although for higher values of train speed $\nu$ the peaks of ground vibrations corresponding to sleeper passage frequencies go out of the frequency band considered, the average level of vibration increases with increase of $\nu$. This is also seen in Fig. 8 which displays the amplitude of $\frac{1}{3}$-oct spectral component of ground vibrations generated by the same TGV or Eurostar trains at the central frequency of $25 \mathrm{~Hz}$ as a function of train speed $\nu$. The sharp peak around $\nu=20 \mathrm{~m} / \mathrm{s}$ relates to the sleeper passage frequencies.

Figure 9 illustrates the ground vibration spectra (in $\mathrm{dB}$, relative to the reference level of $10^{-9} \mathrm{~m} / \mathrm{s}$ ) generated by TGV or Eurostar trains consisting of $N=5$ equal carriages 


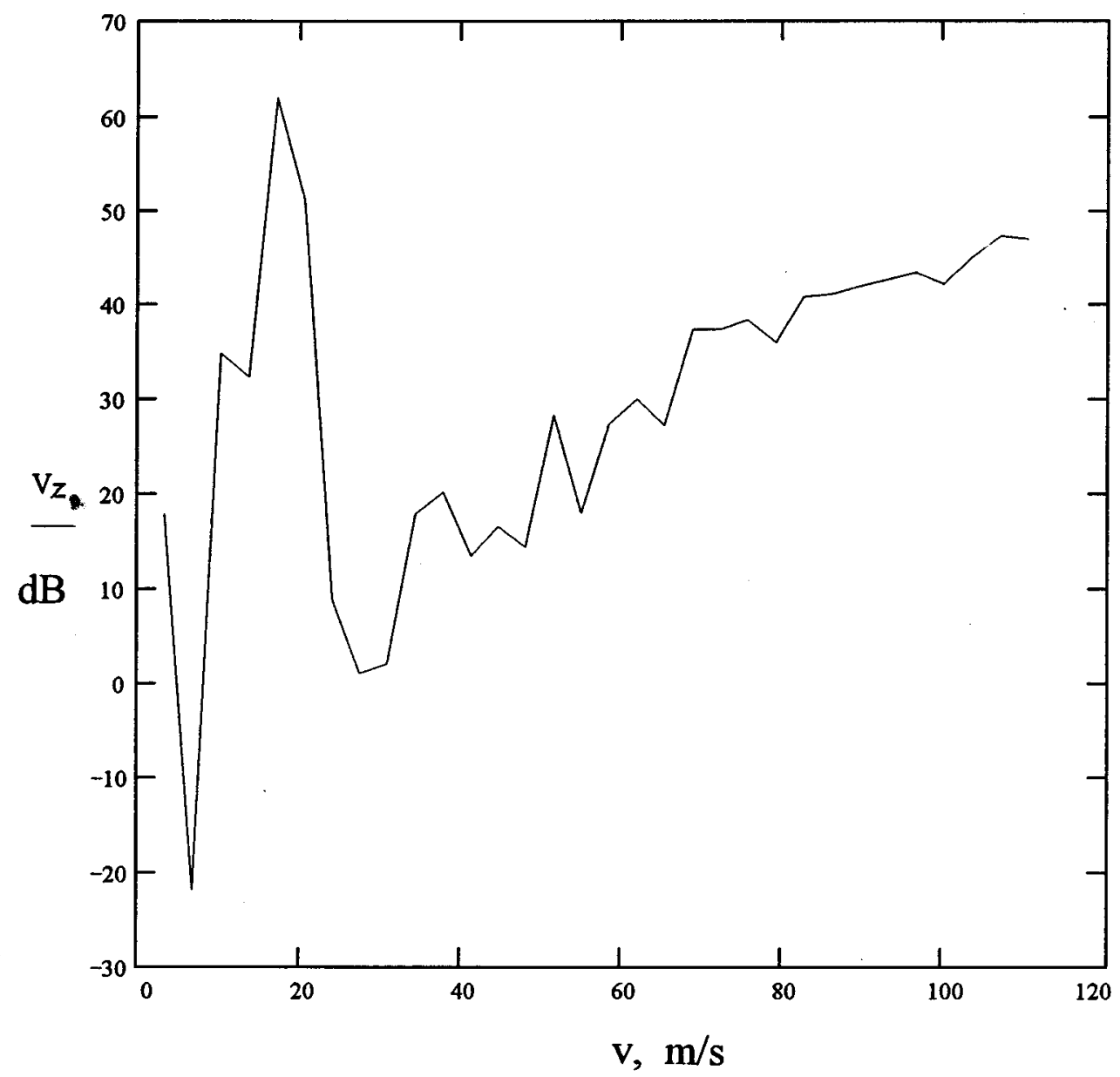

FIG. 8. Amplitude of $\frac{1}{3}$-octave component of ground vibration spectra (in $\mathrm{dB}$, relative to the reference level of $10^{-9} \mathrm{~m} / \mathrm{s}$ ) generated by TGV or Eurostar trains consisting of $N=5$ equal carriages at the central frequency of $25 \mathrm{~Hz}$ as a function of train speed $\nu$.

for both sub-Rayleigh and trans-Rayleigh train speeds: respectively, $\nu=50 \mathrm{~km} / \mathrm{h}$ (curve Vz1) and $\nu=500 \mathrm{~km} / \mathrm{h}$ (curve Vz2). The Rayleigh wave velocity in the ground was $c_{R}=125 \mathrm{~m} / \mathrm{s}(450 \mathrm{~km} / \mathrm{h})$, the critical track wave velocity had a value $c_{\min }=326 \mathrm{~m} / \mathrm{s}(1173.6 \mathrm{~km} / \mathrm{h})$, and the soil attenuation coefficient was $\gamma=0.05$. Other parameters used in calculations were $T=100 \mathrm{kN}, \beta=1.28 \mathrm{~m}^{-1}$, and $y_{0}=30 \mathrm{~m}$.

One can see that the averaged ground vibration level from a train moving at trans-Rayleigh speed $500 \mathrm{~km} / \mathrm{h}(138.8$ $\mathrm{m} / \mathrm{s}$ ) is approximately $70 \mathrm{~dB}$ higher than from a train traveling at speed $50 \mathrm{~km} / \mathrm{h}(13.8 \mathrm{~m} / \mathrm{s})$. This very large increase in ground vibration level agrees well with the general analytical estimates given in the previous sections and with the numerical results obtained for a single-axle load (Figs. 5 and 6).

Influence of soil attenuation on ground vibration spectra generated by TGV or Eurostar trains traveling at speed 500 $\mathrm{km} / \mathrm{h}$ is shown in Fig. 10 for three values of attenuation coefficient: $\gamma=0.005$ (curve Vz1), $\gamma=0.015$ (curve Vz2), and $\gamma=0.045$ (curve Vz3). Other parameters are the same as in Fig. 9.

According to Fig. 10, for very low soil attenuation $(\gamma$ $=0.005$ ) the peak levels of ground vibrations generated by trans-Rayleigh TGV trains can be as high as $140 \mathrm{~dB}$ (relative to $10^{-9} \mathrm{~m} / \mathrm{s}$ ). This corresponds to ground vibration velocities of about $10 \mathrm{~mm} / \mathrm{s}$. Vibrations of such a high level may even cause damage to nearby properties.

Finally, Fig. 11 illustrates the effect of reducing the critical track wave velocity $c_{\min }$, e.g., by making use of softer or thinner ballasts, on generating ground vibration spectra by TGV or Eurostar trains comprising five equal carriages and traveling at speed $500 \mathrm{~km} / \mathrm{h}$. Calculations were carried out for $c_{\min }=326 \mathrm{~m} / \mathrm{s}$ (curve Vz1) and $c_{\min }=140 \mathrm{~m} / \mathrm{s}$ (curve Vz2); other parameters were the same as in Fig. 10. One can see that a lower critical track wave velocity corresponds to lower values of generated ground vibration spectra, in agreement with the results obtained for a single moving load (Fig. 6). Thus using appropriate artificial reduction of minimal track wave velocities, e.g., by applying softer ballast or rubber pads, one could significantly reduce the ground vibration levels.

\section{Discussion}

One must keep in mind that, although taking into account effects of track dynamics, the model used in this paper remains highly idealized. Here we discuss some possible reasons why the predicted ground vibration spectra from highspeed trains may differ from those observed. 


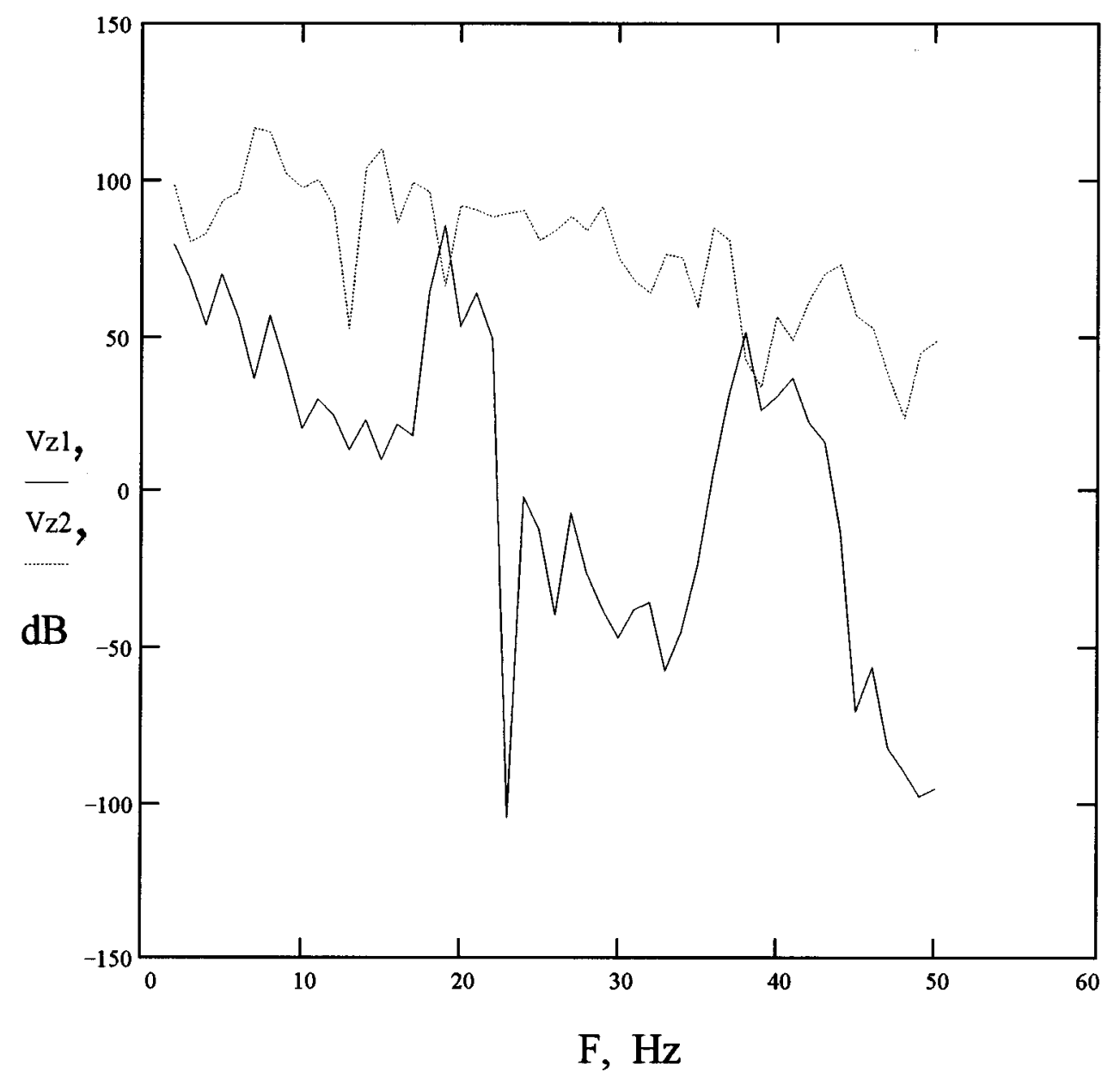

FIG. 9. Ground vibration spectra (in $\mathrm{dB}$, relative to the reference level of $10^{-9} \mathrm{~m} / \mathrm{s}$ ) generated by TGV or Eurostar trains consisting of $N=5$ equal carriages for both sub-Rayleigh and trans-Rayleigh train speeds, respectively: $\nu=50 \mathrm{~km} / \mathrm{h}$ (curve Vz1) and $\nu=500 \mathrm{~km} / \mathrm{h}$ (curve Vz2).

First of all, we did not consider influence of a layered structure of the ground which is present in most practical situations. We recall that in layered media Rayleigh waves become dispersive, i.e., their phase velocity $c_{R}$ is a function of frequency: $c_{R}=c_{R}(\omega)$. Since a shear modulus of the ground $\mu$ normally has higher values at larger depths, this will cause an increase of Rayleigh wave velocities at lower frequencies associated with deeper penetration of surface wave energy into the ground. This may violate the transRayleigh condition $\nu>c_{R}$ and cause significant reduction in low-frequency components of ground vibration spectra generated by high-speed trains. The problem of vibrational impact of high-speed trains on layered grounds deserves special attention and will be considered in a separate paper. Meanwhile, we point out that the model of a homogeneous elastic half-space, used above, is applicable for practical situations if the upper layers have thickness larger than the Rayleigh wavelength at given frequency. For example, if the upper layer of thickness $10 \mathrm{~m}$ is characterized by shear wave velocity of $140 \mathrm{~m} / \mathrm{s}$, the model of a homogeneous half-space is applicable for frequencies above $11 \mathrm{~Hz}$.

Another possible reason why the predicted very large increase of $70 \mathrm{~dB}$ may not be observed is that the wheel-axle pressure mechanism of ground vibration generation may not dominate in the present normal rolling stock. If other pos- sible generation mechanisms mentioned in Sec. I A do prevail, then the expected averaged increase in ground vibration level should be reduced roughly down to $(70-I) \mathrm{dB}$, where $I=20 \log \left(A_{\text {oth }} / A_{\text {wp }}\right)$ determines the relation (in $\mathrm{dB}$ ) between the ground vibration amplitudes due to the wheel-pressure mechanism $\left(A_{\mathrm{wp}}\right)$ and other $\left(A_{\mathrm{oth}}\right)$ mechanisms of generation.

Finally, it should be mentioned that we did not consider possible influence of bulk compressive and shear elastic waves ( $P$ and $S$ waves), radiated into the bulk of the ground, on the total level of ground vibrations generated by highspeed trains. Obviously, radiated $P$ and $S$ waves can also be significantly amplified if the train speeds are high enough so that the conditions $\nu>c_{t}$ or even $\nu>c_{l}$ hold, in addition to the trans Rayleigh condition $\nu>c_{R}$ considered so far (we recall that $c_{R}<c_{t}<c_{l}$ ). In such cases these waves will be radiated into the ground as conical Mach waves propagating at the angles $\Theta_{t}=\arccos \left(c_{t} / \nu\right)$ and $\Theta_{l}=\arccos \left(c_{l} / \nu\right)$ relative to the track, in addition to Rayleigh waves radiated as quasiplane waves along the surface at the angles $\Theta=\arccos \left(c_{R} / \nu\right)$.

From the point of view of radiated energy, all these waves, being dependent on work done by axle loads on track/ground deformation, are not related to each other. This is why the increase in amplitudes of one type of wave due to changing train velocity will not affect the others. A certain 


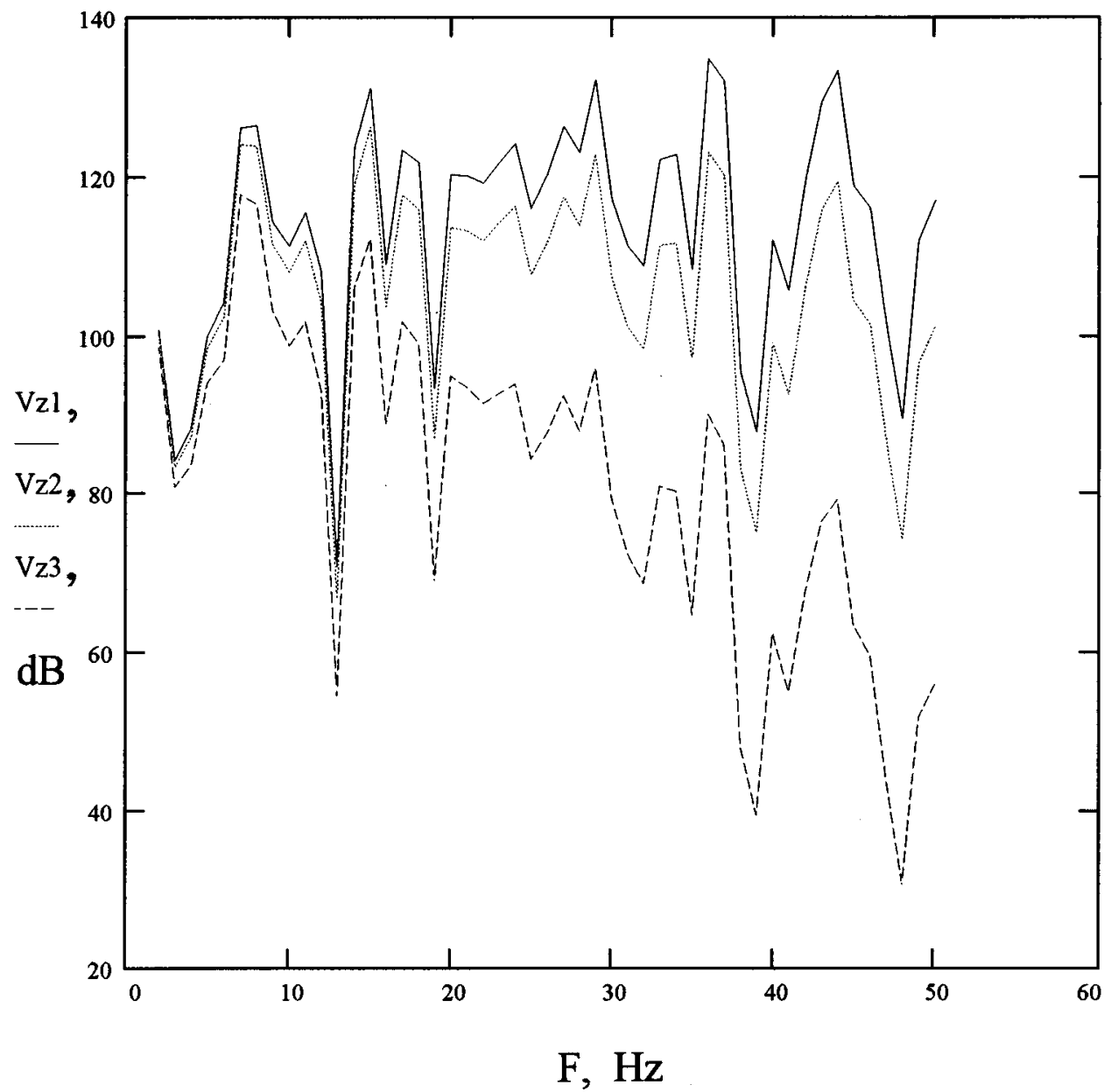

FIG. 10. Effect of soil attenuation constant $\gamma$ on ground vibration spectra (in $\mathrm{dB}$, relative to the reference level of $10^{-9} \mathrm{~m} / \mathrm{s}$ ) generated by TGV or Eurostar trains comprising $N=5$ equal carriages and traveling at trans-Rayleigh speed $\nu=500 \mathrm{~km} / \mathrm{h}$. The results are shown for $\gamma=0.005$ (curve Vz1), $\gamma=0.015$ (curve Vz2), and $\gamma=0.045$ (curve Vz3).

theoretical possibility of the influence of radiated bulk waves on generating Rayleigh waves may be related to the fact that all these waves cause influence on the rolling friction applied to the train. The increase of radiated wave energy due to the opening of additional radiation channels (conical $P$ and $S$ waves) may result in an increase of rolling friction, thus limiting train speed, and hence, the amplitudes of all generated waves (if additional locomotive power is not involved). Such influence, however, seems not to be essential in comparison with possible direct contribution of radiated bulk waves to the total ground vibration field.

The most likely contribution might be that of radiated $S$ waves since their velocity $c_{t}$, being only about $10 \%$ higher than the velocity of Rayleigh waves, can be more easily achieved by moving trains than the velocity of $P$ waves $c_{l}$. In the presence of a layered structure in the ground, the $S$ waves initially radiated into the bulk at the angles $\Theta_{t}=\arccos \left(c_{t} / v\right)$ relative to the track, may experience total internal reflection on the layer boundaries and return to the surface, thus contributing to the total ground vibration field. Repeated reflections from the ground surface and from the layer bottom may cause a waveguide propagation of $S$ waves that may affect the vibration field in relatively remote locations. Another possible influence of effectively generated shear waves on the total ground vibration field may occur for high-speed underground trains. In this case the contribution of bulk shear waves is often more essential than that of Rayleigh waves. ${ }^{22}$ Both of these cases, however, need special attention and will be considered elsewhere.

\section{CONCLUSIONS}

High-speed trains are generally accompanied by increased levels of generated ground vibrations. An especially large increase in ground vibration amplitudes occurs for trans-Rayleigh trains, i.e., for trains traveling at speeds larger than Rayleigh wave velocity in the ground. Calculations performed for French TGV or Eurostar trains show that the average increase of about $70 \mathrm{~dB}$ may take place as compared with conventional trains.

The effect of track bending waves may cause noticeable reduction in amplitudes of ground vibrations generated by trans-Rayleigh trains if train speeds approach the minimal phase velocity of track bending waves. Note that minimal phase velocities of bending waves propagating in the system track/ground are usually much higher than speeds achieved by modern high-speed trains. Nevertheless, using appropriate artificial reduction of minimal track wave velocities in the 


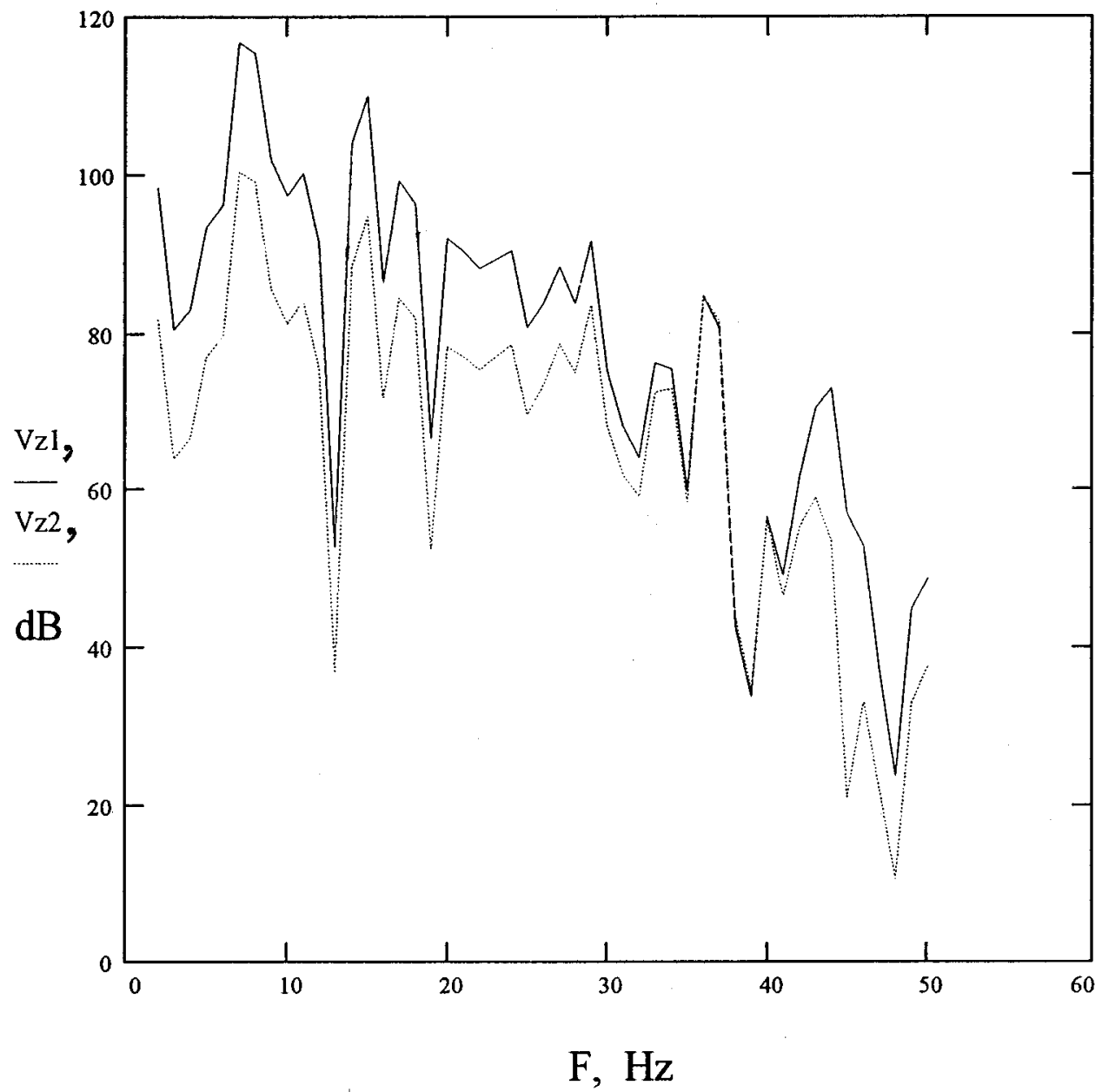

FIG. 11. Effect of reduction of the critical track wave velocity $c_{\min }$ on ground vibration spectra (in $\mathrm{dB}$, relative to the reference level of $10^{-9} \mathrm{~m} / \mathrm{s}$ ) generated by TGV or Eurostar trains comprising $N=5$ equal carriages and traveling at trans-Rayleigh speed $\nu=500 \mathrm{~km} / \mathrm{h}$. The results are shown for $c_{\min }=326 \mathrm{~m} / \mathrm{s}$ (curve Vz1) and $c_{\min }=140 \mathrm{~m} / \mathrm{s}$ (curve Vz2).

most vulnerable parts of the train rout, e.g., by applying softer ballasts or rubber pads, one could reduce generated ground vibrations.

Although trans-Rayleigh trains can generate very intensive ground vibrations, this may not happen everywhere. Fortunately, soils with comparatively low Rayleigh wave velocities (around $100 \mathrm{~m} / \mathrm{s}$ ) are uncommon, the most typical range of $c_{R}$ values being $250-500 \mathrm{~m} / \mathrm{s}$. Nevertheless, the designers and builders of tracks for high-speed trains should be aware of the potential risk of excessive ground vibrations associated with such trains.

\section{ACKNOWLEDGMENTS}

I thank Professor C. C. Ferguson for useful discussions and suggestions. I am also very grateful to the referees of the paper for their very competent comments.

${ }^{1}$ D. Banister and P. Hall, "The second railway age," Built Environment 19, 157-162 (1994)

${ }^{2}$ W. C. Streeter, "The French train a grand vitesse," Built Environment 19, 184-202. (1994).

${ }^{3}$ P. J. Remington, L. G. Kurzweil, and D. A. Towers, Transportation Noise. Reference book, edited by Paul Nelson (Butterworths, London, 1987).

${ }^{4}$ T. M. Dawn, "Ground vibrations from heavy freight trains," J. Sound Vib. 87, 351-356 (1983).
${ }^{5}$ J. Melke, "Noise and vibration from underground railway lines: Proposals for a prediction procedure,"' J. Sound Vib. 120, 391-406 (1988).

${ }^{6}$ R. A. J. Ford, "Inhibiting the transmission of ground-borne vibrations by placing masses on the surface of the ground,"' The Institution of Engineers Australia Vibration and Noise Conference, Melbourne, 1990, pp. $227-$ 231.

${ }^{7}$ C. J. C. Jones, "Use of numerical models to determine the effectiveness of anti-vibration systems for railways," Proc. Inst. Civ. Eng. Transp. 105, 43-51 (1994).

${ }^{8}$ V. V. Krylov and C. C. Ferguson, "Calculations of ground vibrations from heavy-freight trains," in Proc. of 1993 Autumn Conference "Environmental Noise," Windermere, November 1993, Proc. Inst. Acoust. 15 (Part 8), 59-68 (1993).

${ }^{9}$ V. V. Krylov and C. C. Ferguson, "Calculation of low-frequency ground vibrations from railway trains," Appl. Acoust. 42, 199-213 (1994).

${ }^{10} \mathrm{~V}$. V. Krylov, "On the theory of railway-induced ground vibrations," J. Phys. IV 4, C5-769-772 (1994).

${ }^{11}$ V. V. Krylov, "Generation of ground vibrations by superfast trains," Appl. Acoust. 44, 149-164 (1995).

${ }^{12}$ A. I. Belzer, Acoustics of Solids (Springer-Verlag, Berlin, 1988).

${ }^{13}$ S. Timoshenko, Strength of Materials (Van Nostrand, New York, 1942), Part 2.

${ }^{14}$ C. A. Brockley, "The influence of track support structure and locomotive traction characteristics on short wavelength corrugations," Wear 153, 315-322 (1992).

${ }^{15} \mathrm{H}$. Lamb, "On the propagation of tremors over the surface of an elastic solid," Philos. Trans. R. Soc. London Ser. A 203, 1-42 (1904).

${ }^{16}$ W. M. Ewing, W. S. Jardetzky, and F. Press, Elastic Waves in Layered Media (McGraw-Hill, New York, 1957).

Victor V. Krylov: Vibrational impact of high-speed trains 
${ }^{17}$ K. F. Graff, Wave Motion in Elastic Solids (Clarendon, Oxford, 1975).

${ }^{18}$ J. E. White, Seismic Waves: Radiation, Transmission and Attenuation (McGraw-Hill, New York, 1965).

${ }^{19}$ T. G. Gutovski and C. L. Dym, "Propagation of ground vibration: a review,' J. Sound Vib. 49, 179-193 (1976).

${ }^{20}$ D. V. Jones and M. Petyt, "Ground vibration in the vicinity of a strip load: a two-dimensional half-space model,', J. Sound Vib. 147, 155-166 (1991).

${ }^{21}$ J. Cole and J. Huth, "Stresses produced in a half plane by moving loads," J. Appl. Mech. 25, 433-436 (1958).

${ }^{22}$ V. V. Krylov, "Theoretical prediction of ground vibrations from underground trains," Proc. Int. Symp. "Euronoise '95," Lyon, France, 1995 (CETIM, Senlis, 1995), Vol. 1, pp. 211-216. 